\title{
ARTICLE
}

Clinical Research

\section{Impact of preoperative prostate magnetic resonance imaging on the surgical management of high-risk prostate cancer}

\author{
Janet Baack Kukreja ${ }^{1}$ - Tharakeswara K. Bathala ${ }^{1}$. Chad A. Reichard ${ }^{1} \cdot$ Patricia Troncoso $^{1} \cdot$ Scott Delacroix ${ }^{2}$. \\ Benjamin Davies $^{3} \cdot$ Scott Eggener ${ }^{4} \cdot$ Marc Smaldone $^{5} \cdot$ Mohummad Minhaj Siddiqui $^{6} \cdot$ Matthew Tollefson $^{7}$. \\ Brian F. Chapin ${ }^{1}$
}

Received: 24 April 2019 / Revised: 20 July 2019 / Accepted: 29 July 2019 / Published online: 9 September 2019

(C) The Author(s), under exclusive licence to Springer Nature Limited 2019

\begin{abstract}
Objective To evaluate the effect of adding multiparametric magnetic resonance imaging (mpMRI) to pre-surgical planning on surgical decision making for the management of high-risk prostate cancer (HRPC).

Patients and methods A survey was designed to query multiple centers on surgical decisions of 41 consecutive HRPC cases seen from 2012 to 2015. HRPC was defined by the National Comprehensive Cancer Center Network guidelines. Six fellowship-trained urologic oncologists were asked for their surgical plan in regards to the degree of planned nerve-sparing and lymph node dissection. Two rounds of surveys were administered to six external urologic oncologists. The first survey included the case description only and the second included case description with mpMRI images and report. The correct surgical plan was analyzed by correlation of the degree of planned surgical excision and consistency with the final pathologic evaluation. A priori, an effect size of $20 \%$ change was used to determine statistical significance, at $p<0.05$.

Results All cases had at least one change to surgical planning after mpMRI review. Forty (98\%) patients had a change in the degree of planned nerve sparing: wider excision in $32 \%$ and increased nerve sparing in $24 \%$. After mpMRI the correct surgical plan change was made in $49 \%$ for the right and left $51 \%$, decreasing the potential for positive margins. Lymph node dissection was altered from standard to extended lymph node dissection in $17 \%$.

Conclusions Although mpMRI is not integrated in guidelines for preoperative planning in HRPC, its use may impact surgical planning, cancer control, and quality of life.
\end{abstract}

Supplementary information The online version of this article (https:// doi.org/10.1038/s41391-019-0171-0) contains supplementary material, which is available to authorized users.

Brian F. Chapin

bfchapin@mdanderson.org

1 University of Texas MD Anderson Cancer Center, Houston, TX, USA

2 Louisiana State University Health Science Center, New Orleans, LA, USA

3 University of Pittsburgh, Pittsburgh, PA, USA

4 University of Chicago, Chicago, IL, USA

5 Fox Chase Cancer Center, Philadelphia, PA, USA

6 University of Maryland, Baltimore, MD, USA

7 Mayo Clinic, Rochester, MN, USA

\section{Introduction}

Men with high-risk prostate cancer have a high likelihood of extraprostatic extension (EPE) including seminal vesicle invasion (SVI), neurovascular bundle invasion, and lymph node involvement. Over 50\% have previously unrecognized satellite lesions on multiparametric magnetic resonance imaging (mpMRI) [1]. There is often an underestimation of tumor burden and a potentially misguided surgical plan with increased possibility of positive margins [2]. MRI has shown promise in surgical planning in liver and breast cancer with improvements in oncological outcomes [3-5].

Preoperative prostate mpMRI is not routinely recommended as standard practice by guidelines for pre-surgical planning in high-risk prostate cancer patients [6]. Current loco-regional staging with computed tomography (CT) scans and digital rectal exams are inadequate for detecting EPE and SVI [7, 8]. mpMRI can be used to determine the extent of local disease in prostate cancer patients, but has 
not been widely adopted for preoperative surgical planning [8-10].

The purpose of this study was to determine the impact of mpMRI on surgical planning for men with high-risk prostate cancer by analyzing survey data from six experienced urologic oncologists based on clinical data alone, followed by the addition of mPMRI prior to planned radical prostatectomy.

\section{Patients and methods}

A standardized approach to evaluation was implemented by a single surgeon (BFC) for the pre-surgical evaluation of highrisk prostate cancer patients at a single tertiary referral center. The study includes 41 consecutive patients who underwent mpMRI prostate and pelvic imaging, followed by robotassisted laparoscopic prostatectomy and extended pelvic lymph node dissection for clinically localized high-risk prostate cancer. No patients in this study received neoadjuvant systemic therapy or were enrolled in clinical trials. Highrisk prostate cancer was defined according to the National Comprehensive Cancer Network (NCCN) definition ( $\geq \mathrm{cT} 3$, prostate-specific antigen (PSA) $>20 \mathrm{ng} / \mathrm{mL}$, or Gleason $\geq 8$ ) [11]. Data were collected in a prospectively maintained database from 2012 to 2015. All patients underwent standard preoperative staging imaging with $\mathrm{CT}$ of the abdomen and pelvis and a bone scan. Institutional review board approval was obtained. The survey was developed and internally tested prior to sending out for official participation.

Six fellowship-trained urologic oncologists agreed to participate in a survey of management for these 41 high-risk prostate cancer cases. Each urologist previously completed a Society of Urologic Oncology fellowship at six different institutions. They were presented with cases and queried regarding surgical management. Case description included age, American Urologic Association symptoms inventory score, Sexual Health Inventory for Men score, family history, PSA level, prostate exam findings, pathology (Gleason score, total cores, and cores positive, millimeter focus of cancer in each core), and prostate volume. The first survey included the case description with clinical data only. Two months later, a second survey was provided, including the case description with mpMRI images and a standardized mpMRI report. The case order was shuffled with a random number generator for the second iteration of the survey. For each survey, the urologic oncologists were asked for their surgical plan in regards to the degree of planned nerve sparing (none, partial, full) on each side, bladder neck sparing (yes vs. no), lymph node dissection (standard, extended), and surgical approach (robotic vs. open). The questions asked on the questionnaire were designed to ascertain to ask questions about current surgical practices.
Careful attention was directed at not influencing answers by only giving narrow response options.

PIRADS (Prostate Imaging Reporting and Data System) scores were not used as all patients had already been diagnosed with significant high-risk prostate cancer.

\section{Imaging}

All patients were imaged on a $1.5 \mathrm{~T}$ GE HealthCare Signa HDx MR scanner (GE Healthcare, Waukesha, WI) using an eight-channel abdominal array coil and endorectal coil (MR Innerva; Medrad, Pittsburgh, PA). Acquisition specifications advanced over the study period, but typical sequences of the MR protocol included smaller field of view axial, sagittal, and coronal fast spin echo T2-weighted imaging, diffusion-weighted imaging (DWI) with $b$ value of 700 and $1000 \mathrm{~s} / \mathrm{mm}^{2}$ apparent diffusion coefficient reconstruction (ADC), and dynamic contrast imaging (DCE), as well as whole pelvis T1-weighted imaging and DWI with ADC reconstruction (Table 1). DCE-MRI was performed after intravenous injection of gadopentetate dimeglumine (Magnevist, Bayer HealthCare Pharmaceuticals) at $0.1 \mathrm{mmol} / \mathrm{kg}$ of body weight at a rate of $3 \mathrm{~mL} / \mathrm{s}$ via a power injector and consists of 29 consecutive acquisitions over $\sim 3.5 \mathrm{~min}$ to fully evaluate the enhancement characteristics of the prostate gland and any lesion. MRI images were stored and reviewed on a standard picture archiving and communication system. For all cases, radiology review was completed by a radiologist with 10 years of experience reading prostate MRI (TKB) and a standardized report including a standardized set of representative images was generated (see Supplement 1 for an example report). The standardized report example can be seen in Supplement 1.

\section{Statistical analysis}

Statistical analysis was performed with SAS 9.4 (Cary, $\mathrm{NC})$. A priori, the number needed for evaluating effect size was used to determine statistical significance; a 20\% change in 36 surgical plans was determined to be statistically significant at $p<0.05$.

For each patient, there were six questions addressing the surgical plan; thus, for each urologist 246 variables were available for analysis, and a total 1476 variables among the 41 cases.

Change to the surgical plan and correlation with final pathology was also investigated. This evaluated if the surgical plan was appropriately based on the findings at final pathology, that is, a patient with right neurovascular bundle invasion who originally did not have the right neurovascular bundle spared prior to the MRI was changed to a right nonnerve-sparing operation and had right EPE, indicating a positive impact of the mpMRI on surgical planning. The 
Table 1 Three plane localizer

\begin{tabular}{|c|c|c|c|c|c|c|c|}
\hline & $\begin{array}{l}\text { Three plane } \\
\text { localizer }\end{array}$ & $\begin{array}{l}\text { Small FOV } \\
\text { T2 (3-plane) }\end{array}$ & $\begin{array}{l}\text { Small FOV } \\
\text { Axial T1 }\end{array}$ & $\begin{array}{l}\text { Small FOV } \\
\text { Axial DWI }\end{array}$ & $\begin{array}{l}\text { Whole pelvis } \\
\text { Axial T1 pre- } \\
\text { contrast }\end{array}$ & $\begin{array}{l}\text { Whole pelvis } \\
\text { Axial DWI }\end{array}$ & $\begin{array}{l}\text { Small FOV } \\
\text { Axial 3D DCE }\end{array}$ \\
\hline Pulse sequence & Gradient echo & $\begin{array}{l}\text { FSE-XL/ } \\
\text { NPW, } \\
\text { EDR, TRF }\end{array}$ & $\begin{array}{l}\text { FSE-XL/ } \\
\text { NPW, } \\
\text { EDR, TRF }\end{array}$ & $\begin{array}{l}\text { Spin echo/ } \\
\text { EDR, EPI }\end{array}$ & $\begin{array}{l}\text { FSE-XL/NPW, } \\
\text { EDR, TRF }\end{array}$ & $\begin{array}{l}\text { Spin echo/ } \\
\text { EDR, EPI }\end{array}$ & $\begin{array}{l}\text { SPGR/ EDR, fast, } \\
\text { multi-phase }\end{array}$ \\
\hline $\mathrm{TR}(\mathrm{ms})$ & 4.768 & 4000 & 660 & 8000 & 660 & 11,000 & 3.7 \\
\hline $\mathrm{TE}(\mathrm{ms})$ & 1.252 & 140 & 9 & 78 & 9 & 64 & 1.7 \\
\hline Flip angle $\left(^{\circ}\right)$ & 30 & 90 & 90 & - & 90 & - & 25 \\
\hline $\mathrm{BW}(\mathrm{kHz})$ & 25 & 19.23 & 245 & 20 & 240 & 42 & \\
\hline FOV $(\mathrm{cm})$ & 40 & 14 & 14 & 14 & 24 & 38 & 26 \\
\hline $\begin{array}{l}\text { Slice } \\
\text { thickness (mm) }\end{array}$ & 5 & 3 & 3 & 3 & 6 & 5 & 3 \\
\hline Spacing (mm) & 2 & 0 & 0 & 0 & 0 & 0 & 0 \\
\hline $\begin{array}{l}\text { Frequency } \\
\text { direction }\end{array}$ & Unswap & $\begin{array}{l}\mathrm{Ax} / \mathrm{Sg}-\mathrm{A} / \mathrm{P} \\
\mathrm{Co}-\mathrm{S} / \mathrm{I}\end{array}$ & $\mathrm{A} / \mathrm{P}$ & $\mathrm{R} / \mathrm{L}$ & $\mathrm{R} / \mathrm{L}$ & $\mathrm{R} / \mathrm{L}$ & $\mathrm{R} / \mathrm{L}$ \\
\hline Matrix & $256 \times 128$ & $256 \times 224$ & $256 \times 224$ & $128 \times 96$ & $256 \times 192$ & $160 \times 96$ & $160 \times 128$ \\
\hline NEX & 2 & 4 & 4 & 2 & 2 & 2 & 1 \\
\hline Echo train length & 1 & 18 & 3 & 1 & 3 & 1 & 1 \\
\hline
\end{tabular}

$F S E$ fast spin echo, $S P G R$ spoiled gradient echo, $E P I$ echo-planar imaging, $N P W$ no phase wrap, $T R F$ tailored radio frequency, $E D R$ extended dynamic range, $T R$ repetition time, $T E$ echo time, $B W$ bandwidths, $F O V$ field of view, $E T L$ echo train length, $N E X$ number of excitations, $C o$ coronal, $A x / S g$ axial/sagittal, $A / P$ anterior/posterior, $R / L$ right/left, $S / I$ superior/inferior

final surgical margins from the patient cases were used to determine the "correct" surgical plan.

The sensitivity and specificity of the mpMRI and final pathology were calculated. Sensitivity and specificity were calculated separately for EPE, SVI, and pathologic T3, and are presented with $95 \%$ confidence intervals.

\section{Results}

Among the 41 cases, mean PSA was $12.3 \mathrm{ng} / \mathrm{mL}$ (interquartile range: 5.2-13.3), clinical stage ranged from T1c to $\mathrm{T} 3 \mathrm{~b}$, and 40 (95\%) were Gleason $\geq 8$ on biopsy (Table 2). Radiology re-review generated only three changes to the initial MRI reads.

All patients had at least one change to their surgical plan by urologists. Overall, there were $528(36 \%)$ changes in the surgical plan. Following mpMRI review, each urologic oncologist changed their surgical plan between $18 \%$ and $48 \%$ of the cases (Table 3 ). However, the overall agreement of surgical plans was $72 \%$ before mpMRI review and $71 \%$ after; most changes were made to increase agreement, and were almost $20 \%$ increase in agreement of the surgical plan for the nerve sparing (Table 4a). There was consensus for the nerve-sparing strategy for each patient ( $\geq 3$ urologists agreed to increase, decrease, or no change to nerve sparing from what was initially planned) in all but $9 \%$. After mpMRI review, the
Table 2 Characteristics of the 41 cases

\begin{tabular}{ll}
\hline Clinical stage & $n(\%)$ \\
\hline T1c & $14(34)$ \\
T2a & $6(15)$ \\
T2b & $8(19)$ \\
T2c & $5(12)$ \\
T3a & $4(10)$ \\
T3b & $4(10)$ \\
Biopsy Gleason score & $n(\%)$ \\
$3+4$ & $2(5)$ \\
$4+3$ & 0 \\
$4+4$ & $23(56)$ \\
$4+5$ & $15(37)$ \\
$5+5$ & $1(2)$ \\
PSA median (1st-3rd quartile) & $8(5-13)$ \\
Pathologic stage & $n(\%)$ \\
T1c & 0 \\
T2a & 0 \\
T2b & 0 \\
T2c & $13(32)$ \\
T3a & $14(34)$ \\
T3b & $14(34)$ \\
\hline
\end{tabular}

consensus was to increase nerve sparing in $20 \%$, decrease in $32 \%$, and no change in $40 \%$ (Table $4 a$ ). 
Forty (98\%) patients had a change in nerve sparing planned by at least one urologist. Three or more urologists

Table 3 Reviewer changes after MRI review

\begin{tabular}{rllllll}
\hline & $\begin{array}{l}\text { Number } \\
\text { of total } \\
\text { changes to } \\
\text { surgical } \\
\text { plan }\end{array}$ & $\begin{array}{l}\text { Percent } \\
\text { change in } \\
\text { surgical } \\
\text { plan }\end{array}$ & $\begin{array}{l}\text { Bladder } \\
\text { neck } \\
\text { changes }\end{array}$ & $\begin{array}{l}\text { Lymph } \\
\text { node }\end{array}$ & $\begin{array}{l}\text { Left } \\
\text { nerve }\end{array}$ & $\begin{array}{l}\text { Right } \\
\text { nerve }\end{array}$ \\
\hline Reviewer 1 & 37 & 18 & 8 & 6 & 11 & 12 \\
Reviewer 2 & 92 & 44.8 & 18 & 10 & 35 & 29 \\
Reviewer 3 & 98 & 47.8 & 26 & 14 & 26 & 32 \\
Reviewer 4 & 69 & 33.7 & 7 & 17 & 27 & 18 \\
Reviewer 5 & 81 & 39.5 & 15 & 14 & 28 & 24 \\
Reviewer 6 & 64 & 31.2 & 6 & 15 & 18 & 25 \\
\hline
\end{tabular}

Table 4a Agreement amongst urologists

\begin{tabular}{ll}
\hline Agreement in operative plan prior to MRI & $72 \%$ \\
Agreement in operative plan after MRI & $71 \%$ \\
$\%$ change by all urologist after mpMRI review & \\
Bladder neck & $11 \%$ \\
Left nerve & $20 \%$ \\
Right nerve & $19 \%$ \\
Lymph nodes & $10 \%$ \\
\hline
\end{tabular}

${ }^{a}$ Although this number seems small when the plan was initially all correct for $72 \%$ of the patients prior to mpMRI review, the agreement of change is only small numbers likely because so many were initially the correct plan agreed on a change for the nerve sparing after viewing the mpMRI for 61 nerves (74\%) (Table 4b). Overall, 58\% of the nerve-sparing variables changed (Table $4 \mathrm{c}$ ). Changes to planned nerve sparing occurred with 157 (32\%) nerves excised when they were originally planned to be spared and $119(24 \%)$ nerves spared that were originally planned for excision. Figure 1 demonstrates the changes in nerve sparing for all 41 cases; there was a large variation preand post-mpMRI image review for each of the cases.

Table 4b Change in nerve sparing after MRI

Consensus to increase or decrease nerve sparing (considered $\geq 3$ urologist with a change in the same direction after MRI review)

No consensus reached

Consensus to increase nerve sparing $\quad 20 \%$

Consensus to decrease nerve sparing $\quad 32 \%$

Consensus to make no change in nerve sparing $40 \%$

Number of changes to surgical plan among the six urologists for each side-specific nerve sparing for each case

$\begin{array}{ll}0 & 3 \\ 1 & 7 \\ 2 & 71 \\ 3 & 17 \\ 4 & 17 \\ 5 & 22 \\ 6 & 15 \\ \end{array}$

For the above, each nerve was considered separately for a total of 82 nerves
Table 4c Change to elements of surgical planning after reviewing mpMRI

\begin{tabular}{|c|c|c|c|c|}
\hline & \multicolumn{4}{|l|}{$N(\%$ change $)$} \\
\hline Change in any nerve sparing & \multicolumn{4}{|l|}{$286 / 492(58)$} \\
\hline Nerves changed to take & \multicolumn{4}{|l|}{$157 / 492(32)$} \\
\hline Nerves changed to spare & \multicolumn{4}{|l|}{$119 / 492(24)$} \\
\hline Change in bladder neck preservation & \multicolumn{4}{|l|}{$37 / 246(15)$} \\
\hline Change in extent of lymph node dissection & \multicolumn{4}{|l|}{$42 / 246(17)$} \\
\hline Would have ordered MRI for this high-risk case & \multicolumn{4}{|l|}{$202 / 246(82)$} \\
\hline $\begin{array}{l}\text { Change in opinion about whether a preoperative } \\
\text { MRI is helpful }\end{array}$ & \multicolumn{4}{|l|}{$25 / 245,(10)$} \\
\hline Change in surgical approach & \multicolumn{4}{|l|}{$4 / 246(2)$} \\
\hline \multicolumn{5}{|l|}{ Number of changes by urologist number } \\
\hline $\begin{array}{l}\text { Number of urologist who made a change after } \\
\text { MRI review }\end{array}$ & Bladder neck & Lymph nodes & Left nerve & Right nerve \\
\hline 0 & 5 & 0 & 1 & 2 \\
\hline 1 & 13 & 11 & 2 & 5 \\
\hline 2 & 10 & 12 & 7 & 4 \\
\hline 3 & 6 & 7 & 8 & 9 \\
\hline 4 & 6 & 9 & 13 & 9 \\
\hline 5 & 1 & 2 & 7 & 8 \\
\hline 6 & 0 & 0 & 3 & 4 \\
\hline
\end{tabular}




\section{Nerve sparing with and without MRI}

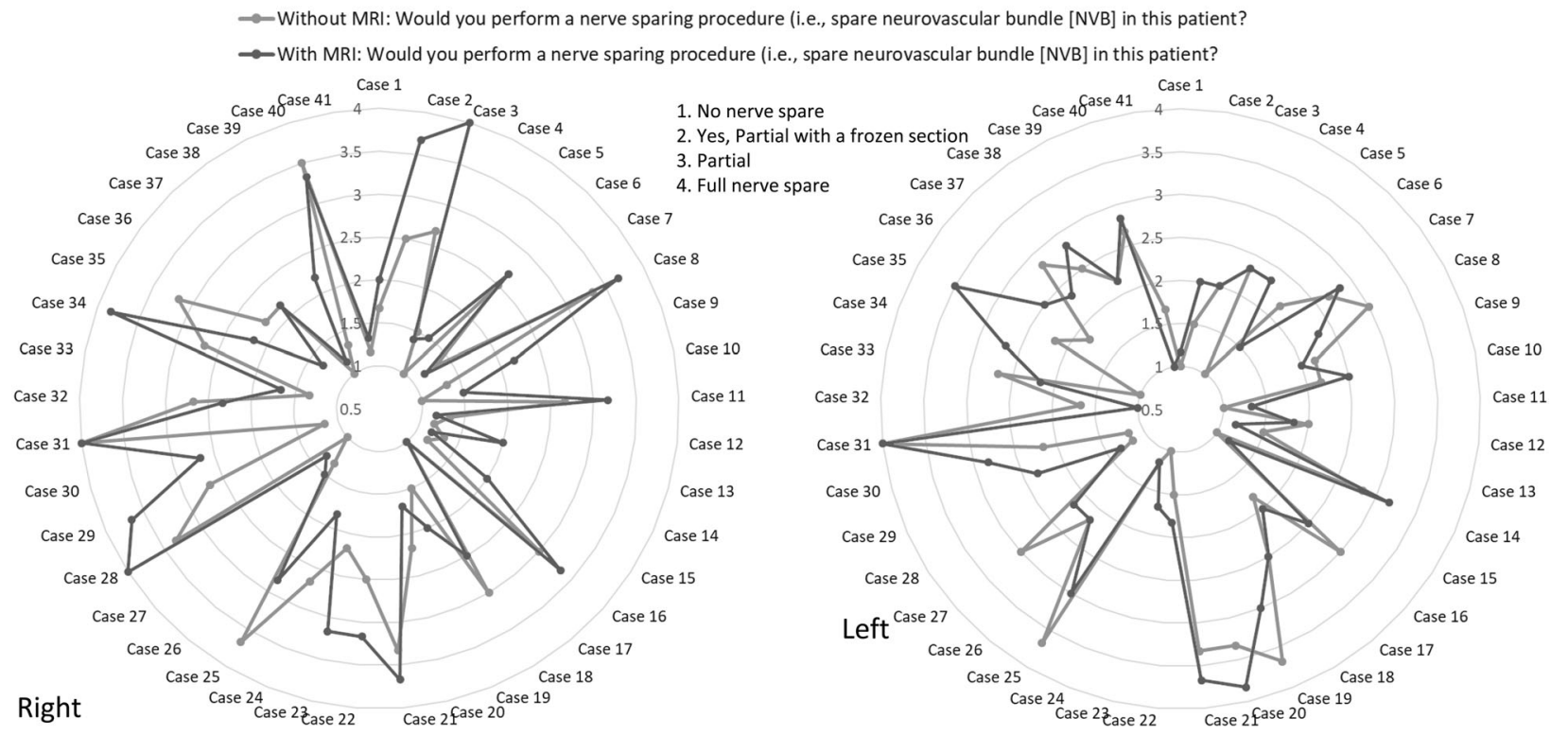

Fig. 1 This figure represents the change of surgical plan after MRI review. Blue color represents the responses when the MRI was not available for review. Orange represents the responses that are the nerve-sparing plans that were made when the MRI was available. The

Table 4d Appropriate surgical plan (degree of surgical excision was consistent with the final pathologic evaluation)

Overall before mpMRI review that were correct

$72 \%$

Overall after mpMRI review that were correct

Overall after mpMRI reivew that were initially correct and $7 \%$ changed to incorrect

After mpMRI review, the following parameters that were initially incorrect were changed to correct

Bladder neck

Right-sided nerve spare

Four cases $(1.6 \%)$ were changed from a robotic to an open approach, all by a single surgeon. Lymph node dissection was altered in 41 cases $(17 \%)$, and 29 changed from standard to extended. Bladder neck sparing was changed 37 times $(15 \%)$ in 30 patients, 32 from sparing to a planned wide bladder neck and 5 from non-sparing to sparing. This change was made by $\leq 2$ urologists $68 \%$ of the time.

Overall, the urologist had the appropriate surgical plan (degree of surgical excision was consistent with the final pathologic evaluation) in $72 \%$ of cases. Overall, after mpMRI review there was a $13 \%$ increase in the overall appropriately planned surgery and a $7 \%$ change to an incorrect surgery. After mpMRI review, the bladder neck sparing was changed to the appropriate surgical plan in $37 \%$ of cases, where the initial plan would have resulted in potentially unfavorable oncologic outcomes. For the right- lines within the circle represent the following: 1 . no nerve sparing, 2 . nerve sparing with a frozen section, 3. partial nerve sparing, and 4. full-nerve sparing

sided nerve sparing, the correct surgical plan change was made in $49 \%$, and for the left-sided nerve sparing, $51 \%$ were changed in those who initially had the potential for positive surgical margins. Values can be found in Table $4 \mathrm{~d}$.

For stage T3a and T3b, mpMRI specificity was $100 \%$ with a positive predictive value (PPV) of $100 \%$ (Table 5). Sensitivity and negative predictive value (NPV) for T3a were 57 and 48\%, respectively. Sensitivity and NPV for T3b were 80 and $90 \%$, respectively.

Urologic oncologists were also queried and $82 \%$ reportedly routinely obtain a mpMRI for surgical planning in men with clinically localized high-risk prostate cancer.

\section{Discussion}

We found urologic oncologist surgical planning for highrisk prostate cancer to be heavily influenced by the findings of a preoperative mpMRI. mpMRI influenced surgical planning to be changed to the "correct" surgical plan in $50 \%$ of the cases. With mpMRI, a more conservative nervesparing approach was taken in $32 \%$ of cases and nerves were spared $24 \%$ of the time, which would otherwise been excised. Our findings suggest that mpMRI may lead to improved outcomes for these high-risk prostate cancer patients. A prospective clinical trial maybe warranted; however, the feasibility of this would be difficult.

Multiple studies in breast cancer have demonstrated a favorable impact of preoperative mpMRI on surgical 
Table 5 Operating characteristics of mpMRI to detect pathologic stage

\begin{tabular}{lllll}
\hline & $\begin{array}{l}\text { Sensitivity }(\%) \\
(95 \% \text { CI })\end{array}$ & $\begin{array}{l}\text { Specificity }(\%) \\
(95 \% \text { CI })\end{array}$ & PPV (95\% CI) & NPV (95\% CI) \\
\hline T3a (EPE) & $57(38-74)$ & $100(70-100)$ & $100(77-100)$ & $48(28-68)$ \\
T3b (SV involvement) & $80(51-95)$ & $100(85-100)$ & $100(70-100)$ & $90(72-97)$ \\
T2 & $100(72-100)$ & N/A & N/A & $100(72-100)$ \\
\hline
\end{tabular}

$E P E$ extraprostatic extension, $S V$ seminal vesicle, $C I$ confidence interval, $P P V$ positive predictive value, $N P V$ negative predictive value, $N / A$ not able to calculate planning and oncological outcomes [12-14]. Importantly, mpMRI is commonly used for preoperative staging to reduce positive surgical margins [15]. Similar to breast mpMRI, preoperative prostate mpMRI has promise in defining the extent of disease, providing more accurate clinical staging, and may help in reducing positive surgical margins or more aggressively preserving erectile nerves.

Recently, mpMRI technology has been used to begin 3D printing of prostate models with their neurovascular bundles, but they have not yet been used in practice for surgical planning [16]. Previous studies have used mpMRI to determine if neurovascular bundle involvement seen on mpMRI correlated with pathologic findings and have found that there is a good correlation [17, 18]. Most studies for mpMRI in the setting of prostate cancer that is not high risk have shown poor operating characteristics for predicting EPE. Although in the setting of high-risk prostate cancer more macro EPE exists, it thus improves the performance of mpMRI compared to micro EPE in prostate cancer that is not high risk. mpMRI is not currently recommended by guidelines prior to surgery despite the correlation with pathologic findings [10]. mpMRI has been found to have value in prostate cancer management, but we have demonstrated its potential added value with regards to operative planning in patients with high-risk prostate cancer [17, 19].

CT scan of the abdomen and pelvis or MRI is recommended by guidelines for staging high-risk prostate cancer [11]. CT provides identification of gross lymphadenopathy, but has limited ability to distinguish normal from abnormal prostate or distinguish NVB involvement with tumor [20]. mpMRI is an approved staging study for prostate cancer and could be applied to more accurately stage patients and potentially improve surgical outcomes [11]. Patients staged with mpMRI have been found to have lower positive surgical margin rates at the time of surgery [21]. Our data suggest that mpMRI in the preoperative setting has a substantial favorable impact on surgical planning. With the ability to evaluate lymph nodes below the aortic bifurcation and improved local prostate staging, mpMRI may reasonably be considered as a standard in the staging of high-risk prostate cancer patients.

The strengths of our study include blinded mpMRI review by an experienced radiologist, generating a standardized report and standardized images. The fellowship-trained urologic oncologists participating, representing six fellowships in the United States, have significant experience with mpMRI and prostate cancer management. Limitations include the lack of generalizability due to the use of one institution's mpMRI protocol (1.5 T with endorectal coil), sequences, and image acquisition along with interpretation by a single genitourinary $(\mathrm{GU})$ radiologist. No $3 \mathrm{~T}$ images were used in this study, which may provide an advantage in prostate cancer detections. The single GU radiologist reviewer may introduce issues of validity and the ability of this study to apply to other institutions. Depending on the radiologist, there may be more consistency locally with a radiologist on EPE compared to across institutions. Lack of consistency could lead to unnecessarily aggressive surgery or incorrect preservation of the neurovascular bundles. If the radiologist is more likely to call EPE, then both the specificity and PPV drops, but sensitivity and NPV would improve. Additional limitations include lack of a control group to evaluate how often surgical plan changed simply by surveying urologist 2 months apart with shuffled case order (i.e., no addition of MRI). All the clinical cases were those of a single clinician at a single institution, which may present additional limitations. We also did not have a control group that did not receive the MRI images, but did receive the report; if this had been part of the study, perhaps, then there may have been better agreement amongst the urologist for surgical plans after the viewing of the report. The interpretation of the MRI on the urologists' part may have led to unnecessary variation. Lastly, since surgeons knew they would subsequently see mpMRIs for the cases, this may have influenced what they reported for their initial management strategy. Thus, if a mpMRI is obtained, it may not provide the useable details for surgical planning that were found in this study.

\section{Conclusion}

mpMRI prior to prostatectomy for high-risk prostate cancer appears to have a significant favorable impact on surgical planning. Preoperative mpMRI may lead to improved decision making regarding nerve-sparing and bladder neck dissection with the potential for decreasing positive surgical margin rates when obtained prior to radical prostatectomy for high-risk prostate cancer. 


\section{Compliance with ethical standards}

Conflict of interest The authors declare that they have no conflict of interest.

Publisher's note: Springer Nature remains neutral with regard to jurisdictional claims in published maps and institutional affiliations.

\section{References}

1. Hegde JV, Margolis DJ, Wang PC, Reiter RE, Huang J, Steinberg ML. et al. Establishing the distribution of satellite lesions in intermediate- and high-risk prostate cancer: implications for focused radiotherapy. Prostate Cancer Prostatic Dis. 2017;20:241-8.

2. Park BH, Jeon HG, Jeong BC, Seo SI, Lee HM, Choi HY. et al. Influence of magnetic resonance imaging in the decision to preserve or resect neurovascular bundles at robotic assisted laparoscopic radical prostatectomy. J Urol. 2014;192:82-8.

3. Bansal GJ, Santosh D, Davies EL. Selective magnetic resonance imaging (MRI) in invasive lobular breast cancer based on mammographic density: does it lead to an appropriate change in surgical treatment?. Br J Radiol. 2016;89:20150679.

4. Marro A, Allen LM, Kives SL, Moineddin R, Chavhan GB. Simulated impact of pelvic MRI in treatment planning for pediatric adnexal masses. Pediatr Radiol. 2016;46:1249-57.

5. Shyn PB, O'Neill AC, Wiggins WF, Sainani NI, Tatli S, Tuncali K, et al. Hepatobiliary phase MRI: impact on planning image-guided liver tumor ablations. Abdom Radiol (NY). 2017;42:2168-74.

6. Maio A, Rifkin MD. Magnetic resonance imaging of prostate cancer: update. Top Magn Reson Imaging. 1995;7:54-68.

7. Saliken JC, Donnelly BJ, Rewcastle JC. The evolution and state of modern technology for prostate cryosurgery. Urology. 2002;60 Suppl 1:26-33.

8. McClure TD, Margolis DJ, Reiter RE, Sayre JW, Thomas MA, Nagarajan R. et al. Use of MR imaging to determine preservation of the neurovascular bundles at robotic-assisted laparoscopic prostatectomy. Radiology. 2012;262:874-83.

9. Johnson DC, Reiter RE. Multi-parametric magnetic resonance imaging as a management decision tool. Transl Androl Urol. 2017;6:472-82.

10. Moore CM, Emberton M. Will the attributes of multiparametric MRI permit the creation of a new approach to therapy?. Curr Opin Urol. 2015;25:518-21.

11. Mohler JL, Armstrong AJ, Bahnson RR, D'Amico AV, Davis BJ, Eastham JA. et al. Prostate cancer, version 1.2016. J Natl Compr Canc Netw. 2016;14:19-30.
12. Brennan ME, McKessar M, Snook K, Burgess I, Spillane AJ. Impact of selective use of breast MRI on surgical decision-making in women with newly diagnosed operable breast cancer. Breast. 2017;32:135-43.

13. Gonzalez V, Sandelin K, Karlsson A, Aberg W, Lofgren L, Iliescu G. et al. Preoperative MRI of the breast (POMB) influences primary treatment in breast cancer: a prospective, randomized, multicenter study. World J Surg. 2014;38:1685-93.

14. Itakura K, Lessing J, Sakata T, Heinzerling A, Vriens E, Wisner D. et al. The impact of preoperative magnetic resonance imaging on surgical treatment and outcomes for ductal carcinoma in situ. Clin Breast Cancer. 2011;11:33-8.

15. Obdeijn IM, Tilanus-Linthorst MM, Spronk S, van Deurzen $\mathrm{CH}$, de Monye C, Hunink MG. et al. Preoperative breast MRI can reduce the rate of tumor-positive resection margins and reoperations in patients undergoing breast-conserving surgery. Am J Roentgenol. 2013;200:304-10.

16. Jomoto W, Tanooka M, Doi H, Kikuchi K, Mitsuie C, Yamada Y. et al. Development of a three-dimensional surgical navigation system with magnetic resonance angiography and a threedimensional printer for robot-assisted radical prostatectomy. Cureus. 2018;10:e2018.

17. Hricak H, Wang L, Wei DC, Coakley FV, Akin O, Reuter VE. et al. The role of preoperative endorectal magnetic resonance imaging in the decision regarding whether to preserve or resect neurovascular bundles during radical retropubic prostatectomy. Cancer. 2004;100:2655-63.

18. Lee T, Hoogenes J, Wright I, Matsumoto ED, Shayegan B. Utility of preoperative 3 Tesla pelvic phased-array multiparametric magnetic resonance imaging in prediction of extracapsular extension and seminal vesicle invasion of prostate cancer and its impact on surgical margin status: experience at a Canadian academic tertiary care centre. Can Urol Assoc J. 2017;11: E174-E8.

19. Hill JR, Fine SW, Zhang J, Eastham JA. Radical prostatectomy for clinical T3 disease: expanding indications while optimizing cancer control and quality of life. Nat Clin Pr Urol. 2007;4:451-4.

20. Heesakkers RA, Hovels AM, Jager GJ, van den Bosch HC, Witjes JA, Raat HP. et al. MRI with a lymph-node-specific contrast agent as an alternative to CT scan and lymph-node dissection in patients with prostate cancer: a prospective multicohort study. Lancet Oncol. 2008;9:850-6.

21. Schiavina R, Bianchi L, Borghesi M, Dababneh H, Chessa $\mathrm{F}$, Pultrone $\mathrm{CV}$, et al. MRI Displays the prostatic cancer anatomy and improves the bundles management before robot assisted radical prostatectomy. J Endourol. 2017;32:315-21. 